\title{
Classification of Avian Pathogenic Escherichia coli by a Novel Pathogenicity Index Based on an Animal Model
}

\author{
Guilherme Fonseca de Souza', Silvio Luís da Silveira Rocha', Thales Quedi Furian', Karen Apellanis Borges', \\ Felipe de Oliveira Salle', Lucas Brunelli de Moraes' ${ }^{2}$, Hamilton Luiz de Souza Moraes' ${ }^{1}$ \& Carlos Tadeu Pippi Salle'
}

\begin{abstract}
Background: Avian Pathogenic Escherichia coli is the main agent of colibacillosis, a systemic disease that causes considerable economic losses to the poultry industry. In vivo experiments are used to measure the ability of E. coli to be pathogenic. Generally, these experiments have proposed different criteria for results interpretation and did not take into account the death time. The aim of this study was to propose a new methodology for the classification of $E$. coli pathogenicity by the establishment of a pathogenicity index based in the lethality, death time and the ability of the strain to cause colibacillosis lesions in challenged animals.
\end{abstract}

Materials, Methods \& Results: A total of 293 isolates of E. coli were randomly selected to this study. The strains were isolated from cellulitis lesions, broiler bedding material or respiratory diseases and were previously confirmed through biochemical profile. The bacterial isolates were kept frozen at $-20^{\circ} \mathrm{C}$. The strains were retrieved from stocks and cultured in brain-heart infusion broth overnight at $37^{\circ} \mathrm{C}$ to obtain a final concentration of $10^{9} \mathrm{UFC} / \mathrm{mL}$. A total of 2940 one-dayold chicks from commercial breeding hens were randomly assigned to groups containing 10 animals and each group was subcutaneously inoculated in the abdominal region with $0.1 \mathrm{~mL}$ of the standard inoculum solution containing each of the strains. A control group of 10 broilers were inoculated with $0.1 \mathrm{~mL}$ of brain-heart infusion broth by the same route. The chicks were kept for seven days. They were observed at intervals of 6,12 and $24 \mathrm{~h}$ post-inoculation during the first days. From the second day on, the chicks were observed at intervals of $12 \mathrm{~h}$. According to the death time and to the scores of each lesion (aerosaculitis, pericarditis, perihepatitis, peritonitis and cellulitis), a formula to determine the Individual Pathogenicity Index was established. A value of 10 was established as the maximum pathogenicity rate for an inoculated bird. From this rate, 5 points corresponded to scores for gross lesions present at necropsy. For each lesion present, it represents 1 point. The remaining 5 points corresponded to the death time. To obtain the death time value, an index of 1, corresponding to the maximum value assigned to a death on the first day, was divided by the number of days that the birds were evaluated, resulting in a value of 0.1428 , which corresponded to a survival bonus factor. It was possible to classify $E$. coli strains into four pathogenicity groups according to the pathogenicity index: high pathogenicity (pathogenicity index ranging from 7 to 10), intermediate pathogenicity (pathogenicity index ranging from 4 to 6.99), low pathogenicity (pathogenicity index ranging from 1 to 3.99) and apathogenic (pathogenicity index ranging from 0 to 0.99 ). The analysis of the strains according to their origin revealed that isolates from broiler bedding material presented a lower pathogenicity index.

Discussion: It is possible that the source of isolation implies in different results, depending on the criteria adopted. This data reinforces the importance of use a more accurate mathematical model to represents the biological phenomenon. In the study, all avian pathogenic Escherichia coli strains were classified based on a pathogenicity index and the concept of the death time represents an interesting parameter to measure the ability of the strain to promote acute and septicemic manifestation. The use of a support method for poultry veterinary diagnostic accompanying the fluctuation of the bacteria pathogenicity inside the farms may indicate a rational use of antimicrobial in poultry industry.

Keywords: APEC, colibacillosis, mathematical model, pathogenicity. 


\section{INTRODUCTION}

Infections with avian pathogenic Escherichia coli (APEC) cause colibacillosis, an acute and systemic disease. Avian colibacillosis is a complex syndrome characterized by multiple organ lesions as aerosacculitis, polyserositis, septicemia and other mainly extraintestinal diseases in chickens, turkeys and other avian species. APECs are found in the intestinal microflora of healthy birds and in the environment. The infection of the respiratory tract by $E$. coli followed for septicemia is referred as an outstanding pathogen for the poultry industry, due to its several economic losses [1,3,6,7,20]. Environmental factors and the poultry immunological system influence the outcome of APEC infections. Thus, in vivo experiments are used to measure the ability of $E$. coli to be pathogenic in poultry [14-17]. One of the first published works on in vivo studies aimed to relate mortality rates in one-dayold chicks [4]. From this work on, other authors have proposed different criteria for results interpretation, although the methodology of the experiments is still similar and did not take into account the death time. Usually, the main parameter assessed by these tests concerns the mortality caused by each strain.

The aim of this study was to propose a new methodology for the classification of $E$. coli pathogenicity by the establishment of a pathogenicity index based in the lethality, death time and the ability of the strain to cause colibacillosis lesions in challenged animals.

\section{MATERIALS AND METHODS}

\section{Experimental animals}

A total of 2940 one-day-old broilers from commercial breeding hens were acquired from a Brazilian poultry company for the study. Birds were randomly assigned to groups containing 10 animals and housed in battery cages that were placed inside an isolation room with controlled temperature $\left(32^{\circ} \mathrm{C}\right)$. Drinking water and commercial feed were provided ad libitum.

Escherichia coli strains and inoculum preparation

A total of 293 strains of Escherichia coli were randomly selected from our stock collection. These strains were isolated from three different broiler sources: cellulitis lesions $(n=162)$, broiler bedding material $(n=$ 72 ) and respiratory diseases $(n=59)$. The strains were previously confirmed as $E$. coli through biochemical profile. The bacterial isolates were kept frozen at $-20^{\circ} \mathrm{C}$ in brain heart infusion broth (BHI) ${ }^{1}$ and glycerol ${ }^{2}$. An amount of $100 \mu \mathrm{L}$ of the culture stocks were retrieved and cultured in $\mathrm{BHI}$ overnight at $37^{\circ} \mathrm{C}$ to obtain a final concentration of $10^{9} \mathrm{UFC} / \mathrm{mL}$. After this period, the inoculum was prepared in aliquots of $0.1 \mathrm{~mL}$ from the cultured BHI.

\section{Inoculation}

Groups of 10 one-day old chicks were subcutaneously inoculated in the abdominal region with 0.1 $\mathrm{mL}$ of the standard inoculum solution containing each of the 293 E. coli strains. A control group of 10 broilers were inoculated with $0.1 \mathrm{~mL}$ of BHI by the same route.

\section{Mortality, evaluation of macroscopic lesions and bacterial reisolation}

The chicks were observed at intervals of 6,12 and $24 \mathrm{~h}$ post-inoculation during the first day. From the second until the seventh day, the chicks were observed at intervals of $12 \mathrm{~h}$. Dead birds were collected for necropsy and for macroscopic evaluation of the presence of the following lesions: pericarditis, perihepatitis, peritonitis, airsacculitis and cellulitis. Chicks that survived until the seventh day were euthanized and necropsied. Animals that died on the first day and that had no lesions were submitted to bacterial isolation from the liver. The material was inoculated in BHI and incubated at $37^{\circ} \mathrm{C}$ for $24 \mathrm{~h}$ and then streaked in eosin methylene blue ${ }^{1}$ and incubated at $37^{\circ} \mathrm{C}$ for $24 \mathrm{~h}$ to observe typical metallic green colonies of E. coli.

\section{Establishment of Individual Pathogenicity Index}

The Individual Pathogenicity Index (IPI) corresponds to the pathogenicity index for each bird in each group inoculated with a strain of E. coli. A value of 10 was established as the maximum pathogenicity rate for an inoculated bird. From this rate, 5 points corresponded to scores for gross lesions present at necropsy. For each lesion present, it represents 1 point. The remaining 5 points corresponded to the death time. To obtain the death time value, an index of 1 , corresponding to the maximum value assigned to a death on the first day, was divided by the number of days that the birds were evaluated, resulting in a value of 0.1428 , which corresponded to a survival bonus factor. Thus, every day that the birds survived was discounted 0.1428 of the death time value. Animals which died on the first day post inoculation and that had positive result on bacterial isolation were presumably died by 
septicemia. Therefore, they received a score 1 for the death time value. According to the death time value and to the scores of each lesion, a formula to determine the IPI was established:

$$
\mathbf{I P I}=(\mathbf{T d} \times \mathbf{5})+\mathbf{P c}+\mathbf{P h}+\mathbf{P t}+\mathbf{A}+\mathbf{C}
$$

Legend: Individual Pathogenicity Index (IPI), death time $(\mathrm{Td})$, pericarditis $(\mathrm{Pc})$, perihepatitis $(\mathrm{Ph})$, peritonitis $(\mathrm{Pt})$, aerosaculitis $(\mathrm{A})$ and cellulitis $(\mathrm{C})$.

Table 1 shows an example of the establishment of the individual pathogenicity index of a strain inoculated.

Table 1. Example of the establishment of the individual pathogenicity index (IPI) of $E$. coli inoculated in 10 one-day old chicks.

\begin{tabular}{cccccccccc}
\hline Strain & Chick & Td & SBF & A & Pc & Ph & Pt & C & IPI \\
\hline 1 & 1 & 1 & 1 & 1 & 1 & 1 & 1 & 0 & 10 \\
1 & 2 & 2 & 0.8572 & 0 & 0 & 1 & 1 & 1 & 7.29 \\
1 & 3 & 2 & 0.8572 & 1 & 1 & 1 & 1 & 1 & 9.29 \\
1 & 4 & 3 & 0.7144 & 1 & 1 & 1 & 1 & 1 & 8.57 \\
1 & 5 & 4 & 0.5716 & 0 & 1 & 1 & 1 & 1 & 6.86 \\
1 & 6 & 4 & 0.5716 & 0 & 0 & 0 & 0 & 0 & 2.86 \\
1 & 7 & E & 0 & 0 & 0 & 0 & 0 & 0 & 0 \\
1 & 8 & E & 0 & 0 & 0 & 0 & 0 & 1 & 1 \\
1 & 9 & E & 0 & 0 & 0 & 0 & 0 & 1 & 1 \\
1 & 10 & E & 0 & 0 & 0 & 0 & 0 & 1 & 1
\end{tabular}

Time of death (Td); survival bonus factor (SBF); aerosaculitis (A); pericarditis (Pc); perihepatitis (Ph); peritonitis (Pt); cellulitis (C); Individual Pathogenicity Index (IPI); euthanized bird at the seventh day (E); lesion present (1); lesion absent (2).

The pathogenicity index for each inoculated strain was calculated according to the equation below:

$$
\mathrm{PI}=\frac{\sum(\mathrm{IPI})}{\mathrm{N}}
$$

Legend: pathogenicity index (PI), sum of individual pathogenicity index $(\Sigma(\mathrm{IPI}))$, total of inoculated chicks in the same group $(\mathrm{N})$

\section{Statistical Analysis}

Data were submitted to one-way analysis of variance and the means were compared by KruskalWallis test, at a $1 \%$ significance level $(P<0.001)$ using SigmaStat software ${ }^{3}$.

\section{RESULTS}

To determine the virulence of the strains after in vivo inoculation, we calculated the death time and we also analyzed the presence of five colibacillosis lesions. There was a significant difference $(P<0.001)$ in the death time among the strains isolated from the three sources. Table 2 describes the death time mean according to the source of isolation.

In the present study, it was possible classify the $E$. coli strains in pathogenicity index as showed in Graphic 1 . There was a predominance of strains with lower indexes ( 0 and 1 ), and a relatively homogeneous distribution among the PIs from 2 to 10 .

Escherichia coli strains were classified into four pathogenicity groups according to the pathogenicity index: high pathogenicity (pathogenicity index ranging from 7 to 10 ), intermediate pathogenicity (pathogenicity index ranging from 4 to 6.99), low pathogenicity (pathogenicity index ranging from 1 to 3.99), apathogenic (pathogenicity index ranging from 0 to 0.99$)$. There is significant difference $(P<0.001)$ among the pathogenicity index mean, as showed in Table 3.

The analysis of results according to the source of isolation, as described in Table 4, revealed significant difference $(P<0.001)$ in their pathogenicity index mean. 
Table 2. Death time according to the source of isolation of E. coli strains.

\begin{tabular}{ccc}
\hline Source of isolation & Total of strains & Death time mean (days) \\
\hline respiratory diseases & 61 & $1.43^{\mathrm{a}}$ \\
cellulitis & 144 & $4.0^{\mathrm{b}}$ \\
broiler bedding material & 59 & $5.7^{\mathrm{b}}$ \\
\hline a,b Different letters in the same column represent significant difference $(P<0.001)$. &
\end{tabular}

Table 3. Classification of 293 Escherichia coli strains in four categories of pathogenicity and their pathogenicity index mean.

\begin{tabular}{ccc}
\hline Classification & Total of strains & Pathogenicity Index (PI) mean \\
\hline High Pathogenicity (H) & 97 & $8.159^{\mathrm{a}}$ \\
Intermediate Pathogenicity (I) & 59 & $4.571^{\mathrm{b}}$ \\
Low Pathogenicity (L) & 103 & $1.685^{\mathrm{c}}$ \\
Apathogenic (A) & 41 & $0.30^{\mathrm{d}}$ \\
\hline a.b,c,d Different letters in the same column represent significant difference $(P<0.001)$.
\end{tabular}

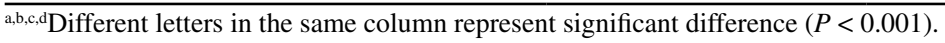

Table 4. Pathogenicity index means and coefficient of variation of E. coli strains according to their source of isolation.

\begin{tabular}{cccc}
\hline Source of isolation & $\begin{array}{c}\text { Total of } \\
\text { strains }\end{array}$ & $\begin{array}{c}\text { Pathogenicity Index } \\
\text { (mean } \pm \text { standard deviation) }\end{array}$ & $\begin{array}{c}\text { Coefficient of } \\
\text { Variation }\end{array}$ \\
\hline respiratory diseases & 59 & $5.420 \pm 2.991^{\mathrm{a}}$ & 55.16 \\
cellutis & 162 & $4.622 \pm 3.245^{\mathrm{a}}$ & 70.21 \\
broiler litter & 72 & $2.319 \pm 2.292^{\mathrm{b}}$ & 98.83 \\
\hline
\end{tabular}

a,b Different letters in the same column represent significant difference $(P<0.001)$.

Graphic 1. Distribution of the 293 Escherichia coli strains according to the Pathogenicity Index.

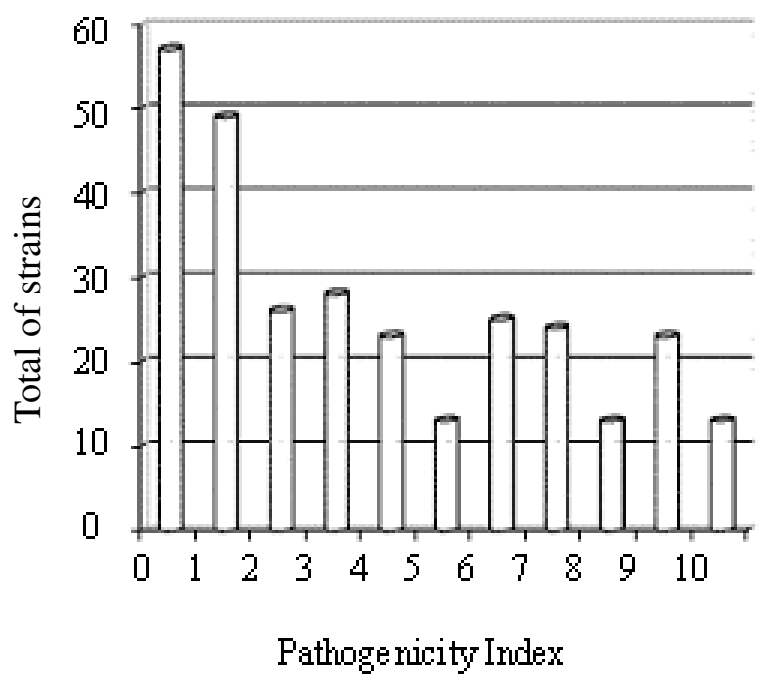




\section{DISCUSSION}

The concept of the death time represents an interesting parameter to measure the ability of the strain to promote acute and septicemic manifestation. Strains isolated of respiratory diseases presented a higher death time mean, reflecting the pathogenic behavior of these strains in the field. Therefore, it is possible that the source of isolation implies in different results, depending on the criteria adopted. This data reinforces the importance of survival bonus factor to use a more accurate mathematical model to represents the biological phenomenon.

The classification in four categories (high pathogenicity, intermediate pathogenicity, low pathogenicity, apathogenic) represents an advancement in $E$. coli studies. It is possible to observe that E. coli isolated from broiler bedding material presented a lower pathogenicity index, and the index was significantly different $(P<0.001)$ of the others sources. Some isolates from cellulites and respiratory diseases showed lower pathogenicity index. Thus, it is likely that in the field, these isolates probably were associated with other factors that provide favorable conditions for bacterial growth and lesions development [1]. On the other hand, some strains isolated from broiler bedding material showed a higher pathogenicity index. Among all the factors contributing to the appearance of lesions, poultry environment is a determining factor and acts directly on changes in the bacterial DNA. The genome of Escherichia coli consists of a conserved part, the so-called core genome, which encodes essential cellular functions and of a flexible, strain-specific part [5]. Genes that belong to the flexible genome code for factors involved in bacterial fitness and adaptation to different environments [5]. It is state that acquisition or loss of genetic information is of great importance for the adaptive evolution of bacteria, which cause disease [9]. Furthermore, the simultaneous acquisition of many genes by horizontal transfer allows transmission of characteristics related to disease in a single step. Thus, these species include pathogenic variants as well as commensal bacteria adapted to different host organisms [19]. In Escherichia coli, various genetic elements encode for pathogenicity factors as well as factors, which increase the fitness of non-pathogenic bacteria [5]. There are approximately $10^{6} \mathrm{CFU}$ of E. coli per gram in avian excreta and $15 \%$ of the strains isolated belong to pathogenic serogroups [8]. Thus, the monitoring of pathogenic E. coli in the poultry farms is important and becomes unavoidable. The difficulty in distinguishing pathogenic and no pathogenic strains of $E$. coli after bacterial isolation is possibly related to the complexity of the interactions that exist among the virulence factors of $E$. coli, beyond the subjectivity of conventional parameters used to determine the pathogenicity [18].

\section{CONCLUSION}

According to the new methodology proposed in this study, it was possible to identify statistically significant differences among the pathogenesis index of strains. The use of the survival bonus factor and the classification of five lesions after the inoculation of chicks reflected the virulence of the strains adequately. Therefore, the use of a support method for poultry veterinary diagnostic accompanying the fluctuation of the bacteria pathogenicity inside the farms may indicate a rational use of antimicrobial in poultry industry.

\section{MANUFACTURERS}

${ }^{1}$ Oxoid. Cambridge, United Kingdom.

${ }^{2}$ Dinâmica. São Paulo, SP, Brazil.

${ }^{3}$ Systat Software. San Jose, CA, USA.

Declaration of interest. The authors report no conflicts of interest and are responsible for the content and writing of the paper.

\section{REFERENCES}

1 Barnes H.J., Nolan L.K. \& Vaillancourt J.P. 2008. Colibacilosis In: Saf Y.M. (Ed). Diseases of Poultry. 12th edn. Ames: Iowa State Press, pp.691-732.

2 Cheung M.K., Li L., Nong W. \& Kwan H.S. 2011. German Escherichia coli O104: H4 outbreak: whole-genome phylogeny whithout alignment. BMC Research Notes. 4(533): 1-5.

3 Dho-Moulin M. \& Fairbrother M. 1999. Avian pathogenic Escherichia coli (APEC). Veterinary Research. 30(2-3): 299-316.

4 Dho-Moulin M. \& Lafont J.P. 1982. Escherichia coli colonization of the trachea in poultry -comparison of virulent and avirulent strains in gnotoxenic chickens. Avian Diseases. 26(4): 787-797. 
5 Dobrindt U., Geddam M., Krumbholz G. \& Hacker J. 2010. Genome dynamics and its impact on evolution of Escherichia coli. Medical Microbiology and Immunology. 199(3): 145-154.

6 Dziva F. \& Stevens M. 2008. Colibacillosis in poultry: unravelling the molecular basis of virulence of avian pathogenic Escherichia coli in their natural hosts. Avian Pathology. 37(4): 355-366.

7 Ferreira A.J. \& Knöbl T. 2009. Colibacilose Aviária. In: Júnior A.B., Silva E.N., Fábio J.D., Sesti L. \& Zuanaze M.A.F. (Eds). Doença das Aves. 2.ed. Campinas: FACTA, p.197-205.

8 Gross W.G. 1994. Disease due to Escherichia coli in poultry. In: Gyles C.L. (Ed.) Escherichia coli in domestic animals and humans. Oxford: Cab International, pp.237-259.

9 Grozdanov L., Raasch C., Schulze J., Sonnenborn U., Gottschalk, G., Hacker J. \& Dobrindt U. 2004. Analysis of the Genome Structure of the Nonpathogenic Probiotic Escherichia coli Strain Nissle 1917. Journal of Bacteriology. 186(16): 5432-5441.

10 Jeong Y.W., Kim T.E., Kim J.H. \& Kwon H.J. 2012. Pathotyping avian Escherichia coli strains in Korea. Journal of Veterinary Science. 13(2): 145-152.

11 Kansal R., Rasko D.A., Sahl J.W., Munson G.P., Roy K., Luo Q., Sheikh A., Kuhne K.J. \& Fleckenstein JM. 2013. Transcriptional Modulation of Enteroxigenic Escherichia coli Virulence Genes in Response to Epithelial Cell Interactions. Infection and Immunity. 81(1): 259-270.

12 Moriel D.G., Rosini R., Seib K.L., Serino L., Pizza M. \& Rappuoli R. 2012. Escherichia coli: Great Diversity around Common a Core. MBio. 3(3): 1-3.

13 Olsen R.H., Christensen H. \& Bisgaard M. 2012. Comparative genomics of multiple plasmids from APEC associated with clonal outbreaks demonstrates major similarities and identifies several potential vaccine-targets. Veterinary Microbiology. 158(3-4): 384-393.

14 Peer F.U., Ansari M.M., Gani I.A. \& Willayat M.M. 2013. Evaluation of inoculation route in the pathogenicity of Escherichia coli strains in broiler chicks. Research Journal for Veterinary Practitioners. 1(2): 18-19.

15 Pourbakhsh S.A., Boulianne M., Martineau-Doizé B., Dozois C.M., Desautels C. \& Fairbrother J.M. 1997. Dynamics of Escherichia coli infection in experimentally inoculated chickens. Avian Diseases. 41(1): 221-233.

16 Raji M.A., Adekeye J.O., Kwaga J.K.P. \& Bale J.O.O. 2003. In vitro and in vivo pathogenicity studies of Escherichia coli isolated from poultry in Nigeria. Israel Journal of Veterinary Medicine. 58(1): 1-15.

17 Sara G.R., Sur S.K., Mitra M., Nag N.C. \& Das A.K. 1995. Colibacillosis in captive birds at Alipur zoo. Indian Journal of Animal Healthy. 34: 43-45.

18 Salle F.O., Fortes F.B.B., Rocha A.C.G.P., Rocha S.L.S., Souza G.F., Moraes H.L.S., Moraes L.B. \& Salle C.T.P. 2010. Utilização de inteligência artificial (redes neurais artificiais) para a classificação do comportamento bioquímico de amostras de Escherichia coli isoladas de frangos de corte. Acta Scientiae Veterinariae. 38(1): 59-62.

19 Touzain F., Denamur E., Médigue C., Barbe V., El Karoui M. \& Petit M.A. 2010. Small variable segments constitute a major type of diversity of bacterial genomes at the species level. Genome Biology. 11(4): 1-15.

20 Tuntufye H.N., Lebeer S., Gwakisa P.S., Goddeeris B.M. 2012. Identification of avian Escherichia coli genes that are induced in vivo during infection in chickens. Applied and Environmental Microbiology. 78(9): 3343-3351. 